\title{
Efficient inhibition of an intraperitoneal xenograft model of human ovarian cancer by HSulf-1 gene delivered by biodegradable cationic heparin-polyethyleneimine nanogels
}

\author{
PING LIU*, MALING GOU*, TAO YI, CHUAN XIE, XIAORONG QI, SHENGTAO ZHOU, \\ HONGXIN DENG, YUQUAN WEI and XIAZHAO
}

\begin{abstract}
Department of Gynecology and Obstetrics, Key Laboratory of Obstetric and Gynecologic and Pediatric Diseases and Birth Defects of Ministry of Education, West China Second Hospital; State Key Laboratory of Biotherapy and Cancer Center, Sichuan University, Chengdu 610041, Sichuan, P.R. China
\end{abstract}

Received April 11, 2011; Accepted June 6, 2011

DOI: $10.3892 /$ or.2011.1550

\begin{abstract}
The HSulf-1 (heparan sulfate 6-O-endosulfatase 1) gene is an important element that modulates the sulfation status of heparan sulfate proteoglycans (HSPGs), leading to the interference of HSPG-related signal transduction pathways. HSulf-1 plays a key role in regulating cell proliferation, tumorigenesis and angiogenesis. Recently, some studies have reported that HSulf-1 is a down-regulated gene in the majority of examined tumor types. In our present study, a recombinant plasmid DNA carrying HSulf-1-cDNA (pHSulf-1) was constructed. The antitumor effect of pHSulf- 1 delivered by heparin-polyethyleneimine (HPEI) nanogels on human ovarian cancer and the possible mechanisms of the antitumor efficacy in vivo were further investigated. Heparin-polyethyleneimine (HPEI) nanogels, as a new safe non-viral gene delivery carrier, were prepared to deliver the plasmid expressing HSulf-1 into HSulf-1-deficient SKOV3 human ovarian cancer cells in vitro and in vivo. pHSulf-1 could be efficiently transfected into SKOV3 ovarian cancer cells by HPEI nanogels in vitro and in vivo. Stable expression of HSulf-1 in vitro and in vivo was verified by reverse transcription polymerase chain reaction (RT-PCR) and Western blot analysis. Furthermore, a SKOV3 intraperitoneal ovarian carcinomatosis model was established to investigate the growth inhibition function of pHSulf-1 in nude mice. Tumor weight was measured. An anti-angiogenesis effect of pHSulf-1 in vivo was detected by CD31 immunostaining and alginate-encapsulate tumor cell assay. Assessment
\end{abstract}

Correspondence to: Professor Xia Zhao, Department of Gynecology and Obstetrics, Key Laboratory of Obstetric and Gynecologic and Pediatric Diseases and Birth Defects of Ministry of Education, West China Second Hospital; State Key Laboratory of Biotherapy and Cancer Center, Sichuan University, No. 20, Section 3, South People's Road, Chengdu 610041, Sichuan, P.R. China

E-mail: xia-zhao@126.com

*Contributed equally

Key words: heparan sulfate 6-O-endosulfatase 1, gene therapy, heparin-polyethyleneimine, ovarian cancer, angiogenesis, apoptosis of apoptotic cells and proliferation index in tumor tissues were performed by TUNEL assay and Ki-67 immunostaining. Intraperitoneal injection of pHSulf-1/HPEI complexes efficiently reduced tumor weight by approximately $87 \%$ compared with control groups $(\mathrm{P}<0.01)$. Meanwhile, reduction in angiogenesis, inhibition of cell proliferation, as well as induction of tumor cell apoptosis were observed, without apparent systemic toxic effects. Collectively, these observations provide the first evidence that pHSulf-1 delivered by HPEI nanogels may become a promising therapeutic strategy against human ovarian cancer.

\section{Introduction}

HSulf-1 has recently been cloned as the human ortholog of QSulf-1. The full-length HSulf-1 cDNA contains an open reading frame of $2616 \mathrm{bp}$ and encodes HSulf-1 protein of 871 amino acids. It has been identified as an extracellular arylsulfatase activity enzyme exhibiting highly specific endoglucosamine-6-sulfatase activity against intact heparin $(1,2)$. Heparan sulfate proteoglycans (HSPGs) play important roles as co-receptors for numerous heparin-binding growth factors, chemokines and adhesion molecules. Thus they may serve as key regulators of cell signaling in the extracellular matrix and on the cell surface (3-5). HSulf-1 can selectively remove the 6-O-sulfate groups from heparan sulfate, resulting in changes in HSPG-related signal transduction pathways, such as fibroblast growth factor 2 (FGF-2), heparin-binding epidermal growth factor (HB-EGF), hepatocyte growth factor (HGF), vascular endothelial growth factor $165\left(\mathrm{VEGF}_{165}\right)$ and Wnt signaling pathways, and consequently dysregulation of HSulf-1 may exert considerable effect on cell growth and tumor formation (6-14). Recently, some studies have identified HSulf-1 as a down-regulated gene in the majority of examined tumor types including ovarian cancer, hepatocellular cancer, breast cancer and head and neck squamous cell carcinoma (6-8). Forced expression of HSulf-1 diminishes signaling of a variety of heparin-binding growth factors, inhibits cell proliferation, migration, invasion and enhances drug-induced apoptosis in vitro (6-10). In addition, re-expression of HSulf-1 
also inhibits angiogenesis, tumorigenesis and promotes druginduced apoptosis in vivo (10-12).

Gene therapy was initially considered as an approach for treating hereditary diseases. Nowadays, its application in cancer treatment has been widely recognized $(15,16)$. At present, many functional genes associated with different tumor types have been identified, but the clinical application of gene therapy is restricted mainly due to lack of safe and efficient gene delivery technologies. Non-viral gene vectors possess many advantages over viral vectors. Most non-viral gene vectors have the capacity of delivering larger DNA molecules, low immunogenicity and they are more cost-effective and convenient to manufacture than viral vectors (17-19). So far, cationic polyethyleneimine (PEI) has been used as one of the most efficient non-viral gene transfection reagents. However, PEI is not biodegradable and has a shortcoming of the correlation among transfection efficiency, cytotoxicity and its chain length $(20,21)$. In order to overcome this limitation, intensive studies have been carried out to couple short PEI chains into a longer one using biodegradable linkers (22-26). Recently, we have synthesized a safe and efficient nonviral gene vector. The low molecular weight PEI was chemically conjugated into biodegradable cationic nanogels by heparin (27).

Ovarian cancer is the leading cause of death among all gynecological malignancies and accounts for $3 \%$ of all cancer types in women (28). Despite improvements in surgery, chemotherapy, radiotherapy and new biological therapies for patients with ovarian cancer, the mortality still remains on a high level (29). Therefore, the development of more feasible, effective and novel therapeutic approaches to combat ovarian cancer is urgently needed.

HSulf-1 is down-regulated in the majority ( $75 \%)$ of tumor tissues originated from ovarian cancer patients (6). In the present study, we used the novel prepared HPEI nanogels which acted as a safe and efficient non-viral gene vector to deliver the plasmid expressing HSulf-1 (pHSulf-1) into HSulf-1-deficient SKOV3 human ovarian cancer cells in vitro and in vivo, with the purpose of exploring the possible mechanism of the tumor suppressor function of HSulf-1 on human ovarian cancer in vivo. Intraperitoneal administration of pHSulf-1/HPEI complexes to the nude mouse bearing intraperitoneal xenograft of human ovarian cancer partly inhibited tumor growth by way of reducing angiogenesis, decreasing cell proliferation and inducing apoptosis. The treatment with pHSulf-1/HPEI complexes was devoid of any conspicuous toxicity.

\section{Materials and methods}

Cell line. The human ovarian serous cystadenocarcinoma cell line SKOV3, obtained from American Type Culture Collection (ATCC, Manassas, VA) was cultured in Dulbecco's modified Eagle's medium (DMEM) supplemented with $10 \%$ heat-inactivated fetal bovine serum, $2 \mathrm{mM}$ L-glutamine, $100 \mathrm{U} / \mathrm{ml}$ of penicillin and $0.1 \mathrm{mg} / \mathrm{ml}$ of streptomycin. Cells were incubated in a humidified atmosphere containing $5 \% \mathrm{CO}_{2}$ at $37^{\circ} \mathrm{C}$ and passaged every 3 days at a split ratio of 1:3 using trypsin.

Construction and purification of plasmid. To insert the HSulf-1 (GenBank accession no. NM-001128205.1 GI:189571640) coding region into the expression plasmid pVAX (Invitrogen), the human skeletal muscle tissue which was confirmed with a higher expression level of HSulf-1 mRNA (1) was collected with the written consent and approval by the Institutional Review Board of Sichuan University. The normal human skeletal muscle tissue sample was acquired from a previously healthy people undergoing surgical resection and snap-frozen in liquid nitrogen immediately. Then the tissue sample was kept at $-80^{\circ} \mathrm{C}$ before the total RNA extraction. Total RNA was extracted using TRIzol reagent (Invitrogen).

The HSulf-1 gene was amplified by reverse transcription polymerase chain reaction(RT-PCR) using Takara one stepRNA PCR kit (Takara, Japan). The expected size of RT-PCR product was $2616 \mathrm{bp}$. The sequences of forward and reverse primer were 5'-CGCGGATCCAAGATGAAGTATTCTTGCTGTGC-3' and 5'-CGCGATATCTTAACCTTCCCATCCATCCCATA-3' for HSulf-1, respectively. The RT-PCR was carried out as follows: reverse transcription $\left(50^{\circ} \mathrm{C}\right.$ for $\left.30 \mathrm{~min}\right)$ and denaturation $\left(94^{\circ} \mathrm{C}\right.$ for $\left.2 \mathrm{~min}\right)$; amplification for 35 cycles $\left(94^{\circ} \mathrm{C}\right.$ for $0.5 \mathrm{~min})$, annealing $\left(60^{\circ} \mathrm{C}\right.$ for $\left.0.5 \mathrm{~min}\right)$ and extension $\left(72^{\circ} \mathrm{C}\right.$ for $3 \mathrm{~min}$ ); followed by a terminal elongation procedure $\left(72^{\circ} \mathrm{C}\right.$ for $\left.10 \mathrm{~min}\right)$. The RT-PCR product was purified from a $1.0 \%$ agarose gel using a gel extraction kit (Qiagen). Then it was digested with BamHI/EcoRV and subcloned into pVAX vector digested with $B a m \mathrm{HI} / E c o \mathrm{RV}$ with T4 DNA ligase (Promega) to generate pHSulf-1. As a control, pVAX plasmid without HSulf-1 cDNA was used as an empty vector (named pEP). Positive colonies of DH5 $\alpha$ Escherichia coli containing pHSulf-1 or pEP were cultured in Luria-Bertani broth containing $50 \mu \mathrm{g} / \mathrm{ml}$ of kanamycin. A broad scale preparation of plasmid DNA was purified using an EndoFree Plasmid Giga kit (Qiagen, Chatsworth, CA). The recombinant pHSulf-1 was confirmed by restriction digestion and DNA sequencing.

Preparation of plasmid/HPEI complexes and transfection in vitro. The SKOV3 cell transfection was carried out using a biodegradable cationic nanogel-HPEI nanogels synthesized at the State Key Laboratory of Biotherapy and Cancer Center as previously described (27). To determine the optimal plasmid/ HPEI ratio $(\mu \mathrm{g} / \mu \mathrm{g})$ for efficient gene delivery, a series of experiments with different plasmid/HPEI ratios transfecting SKOV3 cells in vitro were performed. We used the recombinant pVAX plasmid coding the green fluorescent protein (GFP) in these experiments and obtained a maximum efficiency of transfection when $2 \mu \mathrm{g}$ plasmid/20 $\mu \mathrm{g}$ HPEI was used (data not shown).

SKOV3 ovarian cancer cells were seeded in 6-well plates at a density of $2 \times 10^{5} /$ well and cultured for $24 \mathrm{~h}$ to reach $80 \%$ confluence. Plasmid (pHSulf-1 or pEP)/HPEI complexes $(2 \mu \mathrm{g}$ plasmid/20 $\mu \mathrm{g}$ HPEI) were prepared in $1 \mathrm{ml}$ DMEM medium without serum. Meanwhile, normal saline (NS) was also used as a control agent. Cells were incubated with pHSulf-1/HPEI complexes, pEP/HPEI complexes, HPEI nanogels or NS for $6 \mathrm{~h}$, and then the medium was replaced by $2 \mathrm{ml}$ DMEM supplemented with $10 \%$ FBS and incubated for an additional $48 \mathrm{~h}$. Afterwards, the cells and the supernatants were collected and used for further assay. All transfections were performed in triplicate.

Expression of HSulf-1 mRNA detected by RT-PCR. The reverse transcription polymerase chain reaction (RT-PCR) was used to verify the stable expression of HSulf-1 mRNA in transfected SKOV3 cells and intraperitoneal tumor tissues. Total RNA was 
extracted from each experimental group using TRIzol reagent (Invitrogen) and then the RNA concentration was detected by spectrophotometric analysis. The RT-PCR reactions with the isolated total RNA $(0.5 \mu \mathrm{g})$ were carried out as above mentioned. After the reactions were completed, each RT-PCR product $(10 \mu \mathrm{l})$ was electrophoresed in a $1.0 \%$ agarose gel.

Western blot analysis. To verify whether HSulf-1 was re-expressed in the SKOV3 cells after transfection with pHSulf-1/HPEI complexes and intraperitoneal tumor tissues, the SKOV3 cells after transfection and the tumor tissue samples from each experimental group were lysed in modified RIPA lysis buffer containing PMSF (1 mM). Protein concentrations of lysates were detected using the Bio-Rad protein assay (BioRad, Hercules, CA). Equal amounts of protein were loaded onto $8 \%$ SDS-PAGE for electrophoresis, transferred to PVDF membranes (Millipore) and immunoblotted with rabbit antihuman polyclonal antibody against HSulf-1 (diluted 1:1000, Santa Cruz Biotechnology). The blots were incubated with the horseradish peroxidase-conjugated secondary antibody and the immunoreactive bands were visualized by chemiluminescence detection. GAPDH was used as the internal standard.

Establishment of the tumor model and treatment in vivo. The animal experiment procedure was reviewed and approved by the Institutional Animal Care and Treatment Committee of Sichuan University. The female athymic BALB/c nude mice (6-8 weeks old, 18-20 g each) were used to establish the intraperitoneal xenograft model of human ovarian cancer on the basis of a previous study in the State Key Laboratory of Biotherapy and Cancer Center (30).

Briefly, SKOV3 cell suspension $\left(5 \times 10^{6}\right.$ cells in $100 \mu \mathrm{l}$ DMEM without serum and antibiotics) was injected subcutaneously in the backs of 4 nude mice. When the diameter of tumors grew up to about $1 \mathrm{~cm}$, tumors were collected, with the necrotic areas excised and then minced into tiny particles with a diameter $\leq 1 \mathrm{~mm}$. Sufficient DMEM medium was added into these tumor particles to reach a final volume of $12 \mathrm{ml}$ and then 24 nude mice were inoculated intraperitoneally with $0.5 \mathrm{ml}$ of the above mixture, respectively. Seven days after the i.p. tumor inoculation, mice were randomly allocated into four groups (six mice/per group): i) untreated, normal saline (NS); ii) $50 \mu \mathrm{g}$ HPEI nanogels alone; iii) $5 \mu \mathrm{g}$ pEP/50 $\mu \mathrm{g}$ HPEI complexes; and iv) $5 \mu \mathrm{g}$ pHSulf-1/50 $\mu \mathrm{g}$ HPEI complexes (volume, $100 \mu \mathrm{l}$ ).

Afterwards, all mice were intraperitoneally administered the above agents every other day for 12 times. The weight of mice, cachexia and other abnormalities were monitored. These mice were sacrificed 3 days after the last intraperitoneal injection. The intraperitoneal tumors were excised and weighed. At the time of sacrifice, each mouse was observed and recorded in terms of ascite volume, the number and location of peritoneally disseminated macroscopic tumors. A part of the tumor tissues were immediately snap-frozen in liquid nitrogen, and then kept at $-80^{\circ} \mathrm{C}$ for further assay. The remaining of tumor tissues were fixed in $10 \%$ formalin $(\mathrm{pH} 7.0)$ and embedded in paraffin.

Alginate-encapsulate tumor cell assay. An alginate-encapsulate tumor cell assay was performed to explore whether the antitumor effect of HSulf-1 involved the inhibition of angiogenesis as previously mentioned $(31,32)$. Briefly, alginate beads containing $1 \times 10^{5}$ SKOV 3 ovarian tumor cells per bead were implanted s.c. into both dorsal sides of the BALB/c nude mice. Mice were treated every other day with i.p. administration of $5 \mu \mathrm{g}$ pHSulf-1/50 $\mu \mathrm{g}$ HPEI complexes, $5 \mu \mathrm{g} \mathrm{pEP} / 50 \mu \mathrm{g}$ HPEI complexes, $50 \mu \mathrm{g}$ HPEI nanogels or $0.9 \% \mathrm{NS}$. The mice were injected intravenously with $100 \mu \mathrm{l}$ of a $100 \mathrm{mg} / \mathrm{kg}$ FITCdextran (Sigma) solution after 12 days. After FITC-dextran injection, alginate beads were photographed after being exposed surgically and then rapidly removed in $20 \mathrm{~min}$. The uptake of FITC-dextran was measured as previously described $(31,32)$.

Immunohistochemistry staining. CD31 and Ki-67 immunostaining for intraperitoneal tumors were carried out with goat anti-mouse CD31 (diluted 1:100; Santa Cruz Biotechnology) and rabbit anti-human Ki-67 antibody (diluted 1:100; Thermo), respectively. Intraperitoneal tumor sections (3-5 $\mu \mathrm{m})$ were first deparaffinized and rehydrated. The antigen retrieval was performed by heating in $10 \mathrm{mM}$ citrate buffer solution ( $\mathrm{pH}$ 6.0) at $120^{\circ} \mathrm{C}$, endogenous peroxidase activity was blocked by $3 \%$ $\mathrm{H}_{2} \mathrm{O}_{2}$ and then non-specific binding of reagents was blocked with normal goat or rabbit serum. Sections were incubated with primary antibody, biotin-conjugated secondary antibody and streptavidin-biotin complex successively. The diaminobenzidine (DAB) peroxide solution was used to observe the immunoreaction and then the cellular nuclei were counterstained with hematoxylin.

In order to determine the microvessel density (MVD), the number of microvessels were counted in the fields having the highest vascular density without necrosis areas of different sections at $\mathrm{x} 100$ magnification. To quantify the proliferation index, the percentage of $\mathrm{Ki}$-67-positive cells was analysed in the random fields without necrosis areas of different sections at a magnification of $\mathrm{x} 400$ (five high-power fields/slide).

Apoptotic analysis. To detect apoptotic cells in intraperitoneal tumor tissues, terminal deoxynucleotidyl transferase-mediated dUTP nick end-labeling (TUNEL) assay was used to analyse the effect of inducing apoptosis by pHSulf-1/HPEI complexes in accordance with the manufacturer's protocol (Promega). Cell nuclei stained with dark green fluorescence were defined as TUNEL-positive nuclei and visualized by fluorescence microcopy. The apoptosis index was analysed in the random fields without necrosis areas of different sections at a magnification of x400 (five high-power fields/slide).

Evaluation of potential side effects. To evaluate the potential side effects and toxicity of pHSulf-1/HPEI complexes, the animal weight was monitored every four days and the relevant indices such as anorexia, diarrhea, skin ulceration or toxic death were observed consecutively during the whole treatment procedure. After sacrifice, various organs (heart, liver, spleen, lung, kidney and brain) were harvested, fixed in $10 \%$ formalin (pH 7.0) and embedded in paraffin. Sections of these tissues (3-5 $\mu \mathrm{m})$ were stained with $\mathrm{H} \& \mathrm{E}$.

Statistical analysis. All numerical values were recorded as means \pm standard error (SE). Statistical analysis was performed with one-way analysis of variance (ANOVA) to determine differences of the experimental data among different groups 
A

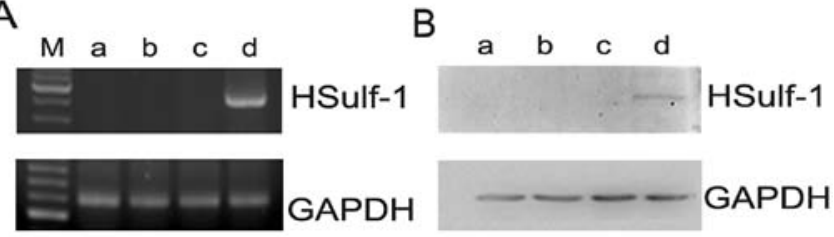

Figure 1. Expression of HSulf-1 by pHSulf-1/HPEI complexes in vitro. SKOV3 ovarian cancer cells were transfected with NS (a), HPEI nanogels (b), pEP/HPEI complexes (c) or pHSulf-1/HPEI complexes (d). (A) PT-PCR showed cells of the four groups had the same positive band at about $500 \mathrm{bp}$ (GAPDH), but only pHSulf-1/HPEI complexes treated cells had positive band at $2616 \mathrm{bp}$ (HSulf-1). (B) HSulf-1 protein was determined by Western blot analysis in SKOV3 cells after transfection. The result showed the positive band $(\sim 130 \mathrm{kDa})$ only occurred in cells transfected with pHSulf-1/HPEI complexes.

using SPSS software. A value of $\mathrm{P}<0.05$ was defined as statistically significant.

\section{Results}

Stable expression of HSulf-1 in SKOV3 cells in vitro. In order to test whether the pHSulf-1/HPEI complexes led to effectively expression of HSulf-1 in SKOV3 ovarian cancer cells by transfection in vitro, SKOV3 cells were seeded in 6-well plates and incubated with pHSulf-1/HPEI complexes, pEP/HPEI complexes, HPEI nanogels and normal saline (NS), respectively. After transfection for $48 \mathrm{~h}$, stable expression of HSulf-1 was detected using PT-PCR and Western blot analysis. HPEI nanogels efficiently transfected pHSulf-1 into SKOV3 ovarian cancer cells in vitro and HSulf-1 was expressed in the SKOV3 cells after transfection with pHSulf-1/HPEI complexes, compared with the control groups (Fig. 1).

Expression of HSulf-1 in intraperitoneal ovarian tumor in nude mice. Next, to examine whether the pHSulf-1/HPEI complexes caused expression of HSulf-1 on SKOV3 cell line in vivo, we established an intraperitoneal xenograft model of human ovarian cancer. The nude mice were treated with the above four agents, respectively. All mice were sacrificed 3 days after the last treatment and the intraperitoneal tumors were collected for RT-PCR and Western blot analysis. The pHSulf-1/HPEI complexes resulted in expression of HSulf-1 in tumor tissues, but no expression of HSulf-1 could be detected in the three control groups (Fig. 2).

pHSulf-1 inhibited intraperitoneal ovarian tumor growth and invasion in nude mice. We established the intraperitoneal xenograft model of human ovarian cancer in nude mice in order to further study the efficacy of pHSulf-1 in inhibiting the growth of human ovarian tumor in vivo. The nude mice were treated with pHSulf-1/HPEI complexes and with pEP/HPEI complexes, HPEI nanogels or normal saline (NS) as control, respectively. The mice were sacrificed 3 days after the last treatment and the intraperitoneal tumors were excised and weighed. The mean tumor weight (Fig. 3) was $0.19 \pm 0.15 \mathrm{~g}, 1.15 \pm 0.26 \mathrm{~g}$, $1.53 \pm 0.32 \mathrm{~g}, 1.42 \pm 0.30 \mathrm{~g}$ in pHSulf-1/HPEI complexes, pEP/ HPEI complexes, HPEI nanogels and normal saline (NS) group, respectively $(\mathrm{P}<0.01)$. No significant difference of the intraperitoneal tumor weight was observed among the control groups.

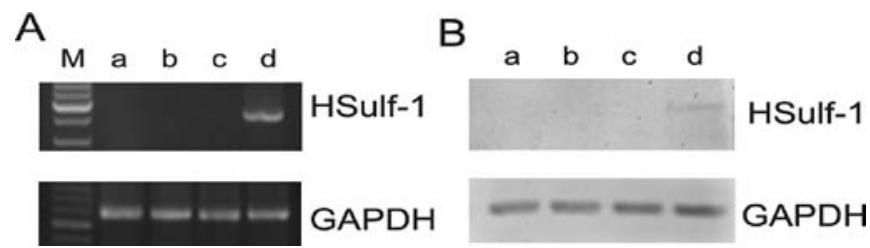

Figure 2. Expression of HSulf-1 by pHSulf-1/HPEI complexes in vivo. The intraperitoneal carcinomatosis model in nude mice was established and intraperitoneally administered with NS (a), HPEI nanogels (b), pEP/HPEI complexes (c) and pHSulf-1/HPEI complexes (d), respectively. (A) PT-PCR showed only the tumor tissues of pHSulf-1/HPEI group had positive band at $2616 \mathrm{bp}$ (HSulf-1). (B) HSulf-1 protein was detected by Western blot analysis in tumor tissues of the four groups and the result showed the positive band $(\sim 130 \mathrm{kDa})$ only occurred in pHSulf-1/HPEI group in contrast to control groups.

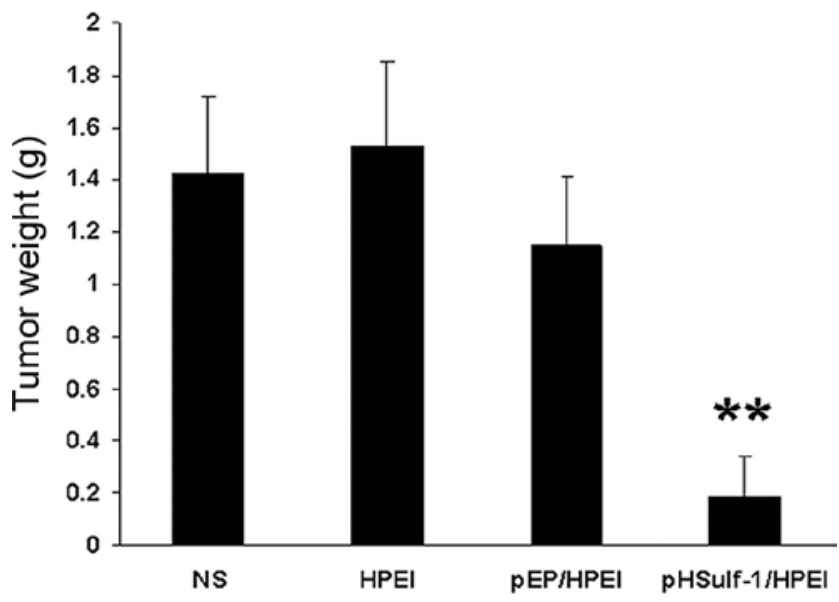

Figure 3. pHSulf-1 significantly inhibited intraperitoneal tumor growth. The intraperitoneal carcinomatosis model in nude mice was established and intraperitoneally administered with pHSulf-1/HPEI complexes, pEP/HPEI complexes, HPEI nanogels and NS, respectively. The mice were sacrificed 3 days after the last treatment and the intraperitoneal tumors were weighed. Data are shown as the means $\pm \mathrm{SE}$. ${ }^{* *} \mathrm{P}<0.01$ vs. NS, HPEI alone and $\mathrm{pEP} / \mathrm{HPEI}$ group, respectively.

Intraperitoneal injection of pHSulf- 1 efficiently reduced tumor weight by about $87 \%$ compared with controls.

In the three control groups, all mice developed macroscopic disseminated tumor nodules intraperitoneally and a portion of them emerged bloody ascites. The tumor nodules were scattered on various viscera and some could even been distinguished by the naked eye like invading the parenchyma of liver instead of only depositing on the surface. Additionally, thickened peritoneum by tumor cells and intestine conglutination were observed in most mice of these groups. Microscopic examination verified that livers of one, two and four of the six mice had tumor invasion, and the bloody ascites emerged in one, three and four of the six mice in pEP/HPEI complexes, HPEI nanogels and normal saline (NS) group, respectively. In the pHSulf-1/HPEI complexes group, the growth of intraperitoneal tumor nodules were limited to the pelvis. No obvious organs and tissues were invaded by the tumor and no ascites were observed in these mice. Two of the six mice exhibited no macroscopic tumor when sacrificed. Intraperitoneal injection of pHSulf-1/HPEI complexes inhibited the invasive capability of ovarian cancer. 
A

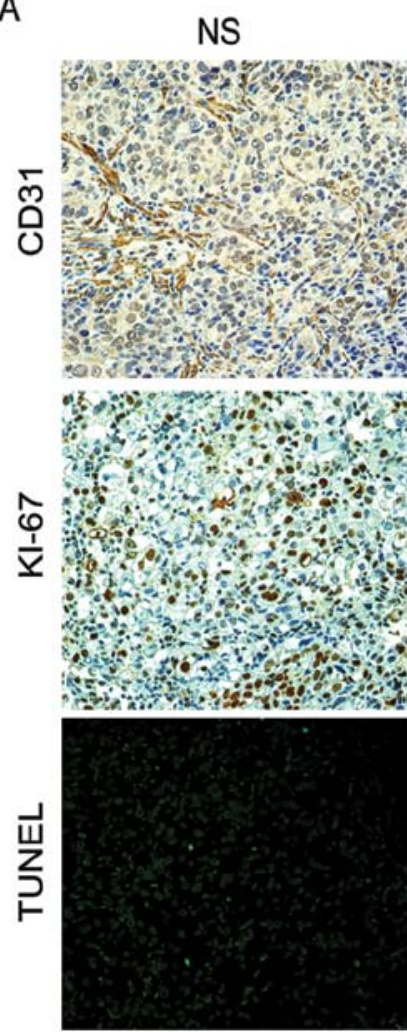

B

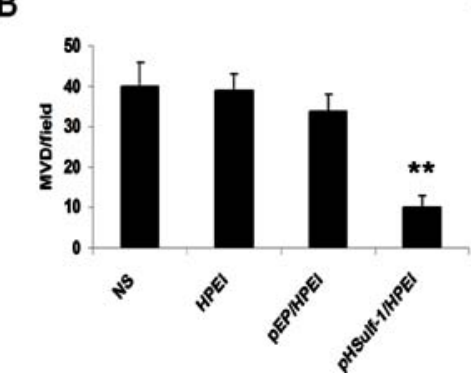

HPEI
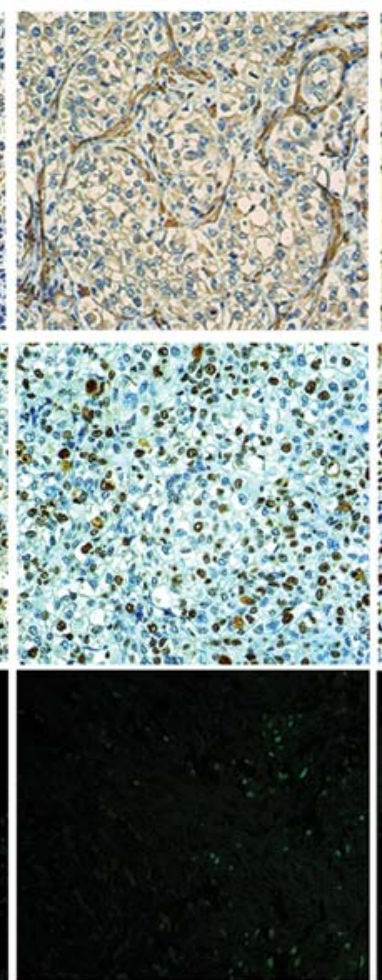

C

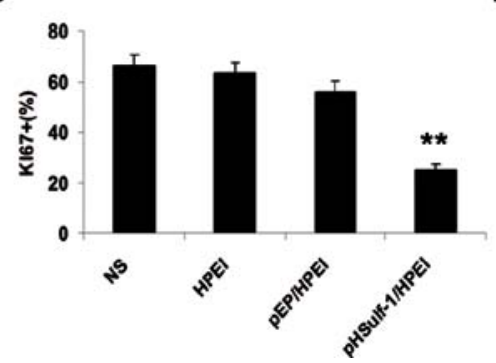

pEP/HPEI
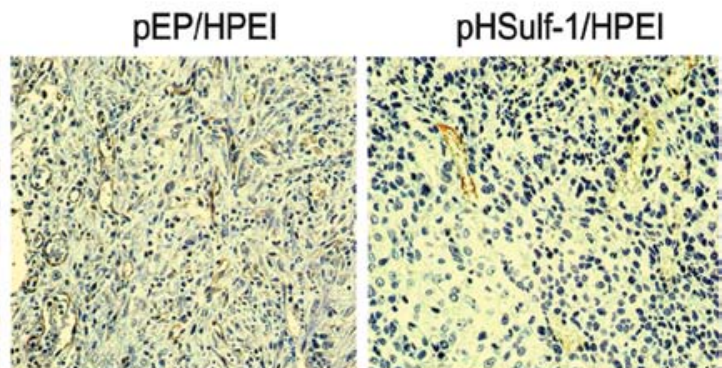

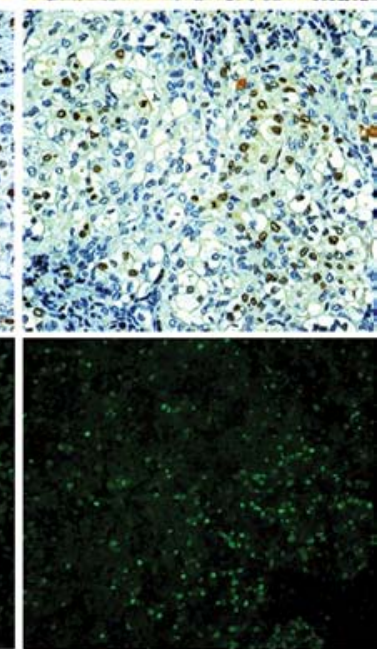

D

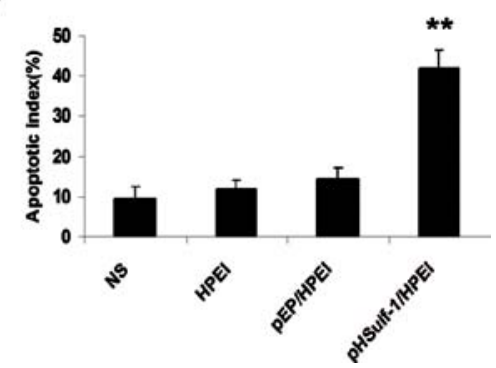

Figure 4. Effects of pHSulf-1 on angiogenesis, cell proliferation and apoptosis in vivo. (A) Top panel, CD31 staining revealed plentiful microvessels in control groups, but few in pHSulf-1/HPEI group; magnification, x400. Middle panel, many Ki-67-positive cells were identified in NS, HPEI and pEP/HPEI groups in contrast to pHSulf-1/HPEI group; magnification, $\mathrm{x} 400$. Bottom panel, TUNEL assay showed only a few positive nuclei in tumor tissues of control groups, but plentiful in pHSulf-1/HPEI group; magnification, $\mathrm{x} 200$. (B) pHSulf-1 significantly reduced CD31-positive microvessels compared with the controls. (C) Percentages of Ki-67-positive nuclei apparently decreased in pHSulf-1/HPEI group. (D) pHSulf-1 markedly increased ( ${ }^{* *} \mathrm{P}<0.01$ ) the percent of apoptosis cells vs. controls. Data are expressed as the means \pm SE.

pHSulf-1 inhibited angiogenesis in vivo. Angiogenesis in tumor tissues was evaluated using CD31 antibody which had high specific affinity of vascular endothelial cells by immunostaining. Brown staining by biotinylation was observed by microscopy. The mean microvessel density (MVD) was obviously reduced in tumors treated with pHSulf-1/HPEI complexes compared with control groups $(\mathrm{P}<0.01$, Fig. $4 \mathrm{~A}$ and B). The capability of anti-angiogenesis was also detected by the alginate-encapsulate tumor cell assay. New blood vessels in alginate beads from the mice treated with pHSulf-1/HPEI complexes were sparse. In addition, FITC-dextran uptake was significantly decreased from this group compared with control groups (Fig. 5A and B).

pHSulf-1 inhibits proliferation and increases apoptosis in vivo. We performed Ki-67 immunostaining to evaluate whether the antitumor efficacy of pHSulf-1 correlated with the decreased tumor cell proliferation. The results indicated that cell proliferation dramatically decreased in tumors treated with pHSulf-1/HPEI complexes compared with control groups $(\mathrm{P}<0.01$, Fig. 4A and $\mathrm{C})$. The number of Ki-67-positive cell nuclei was counted as a ratio of the immunoreactive positive cells to the whole number of cells counted.

The TUNEL assay displayed many strongly positive nuclei in the tumor tissues of pHSulf-1/HPEI complexes group, but such nuclei were rare in tumor tissues of control groups (Fig. 4A). Apoptotic index showed pHSulf-1/HPEI complexes resulted in a significant increase of apoptotic cells vs. control groups $(\mathrm{P}<0.01$, Fig. 4D).

Toxicity observation. The animal weight, which was considered as a parameter for evaluation of physical status, anorexia 

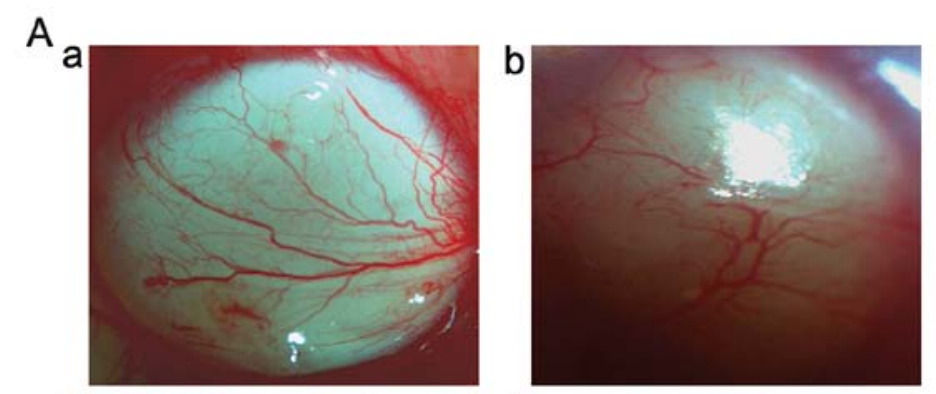

B
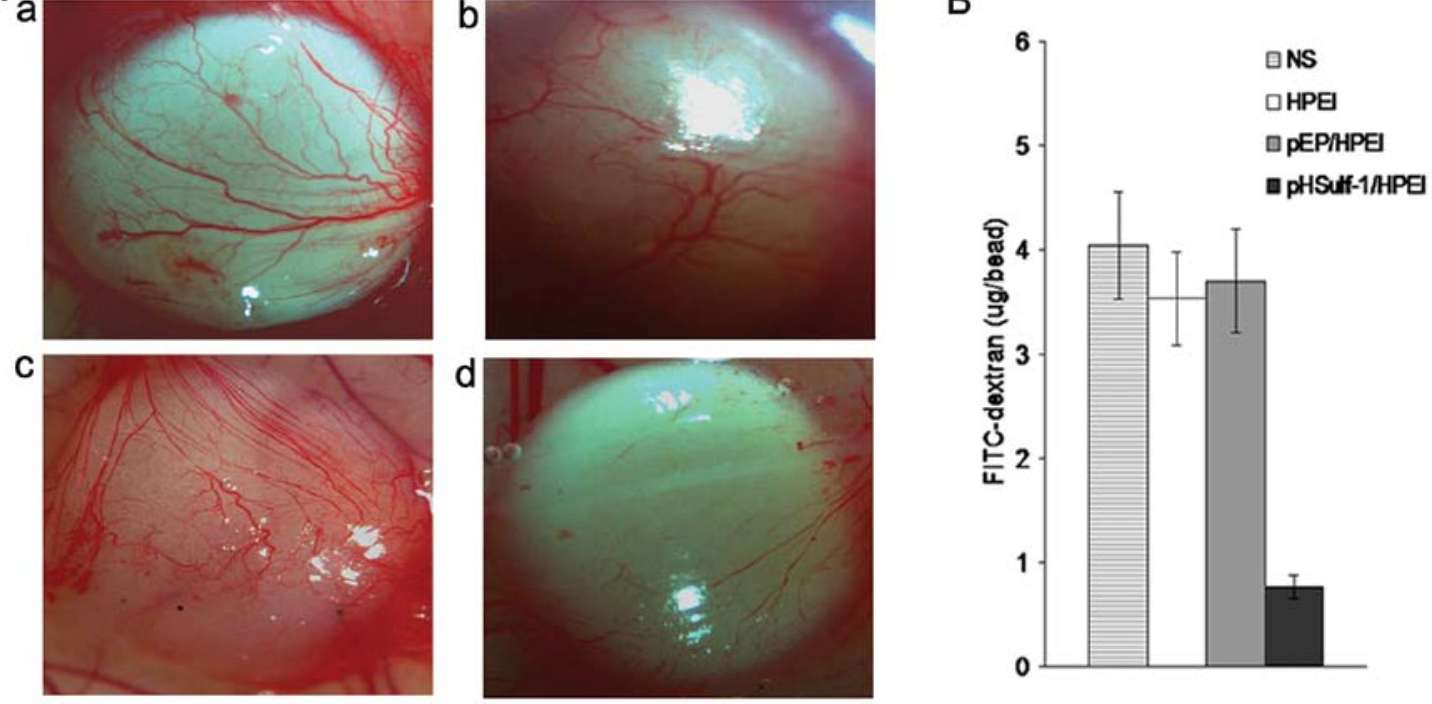

Figure 5. Vascularization of alginate implants. Alginate beads containing $1 \times 10^{5} \mathrm{SKOV} 3$ ovarian tumor cells per bead were implanted s.c. into the backs of the $\mathrm{BALB} / \mathrm{c}$ nude mice. Mice were intraperitoneally administered with NS (a), HPEI nanogels (b), pEP/HPEI complexes (c) or pHSulf-1/HPEI complexes (d) every other day for 12 days. (A) Beads were surgically removed and photographed. (B) FITC-dextran was quantified. FITC-dextran uptake of beads from pHSulf-1/ HPEI treated mice indicated a significant decrease $\left({ }^{* *} \mathrm{P}<0.01\right)$ compared with control groups. Data are expressed as the means $\pm \mathrm{SE}$.

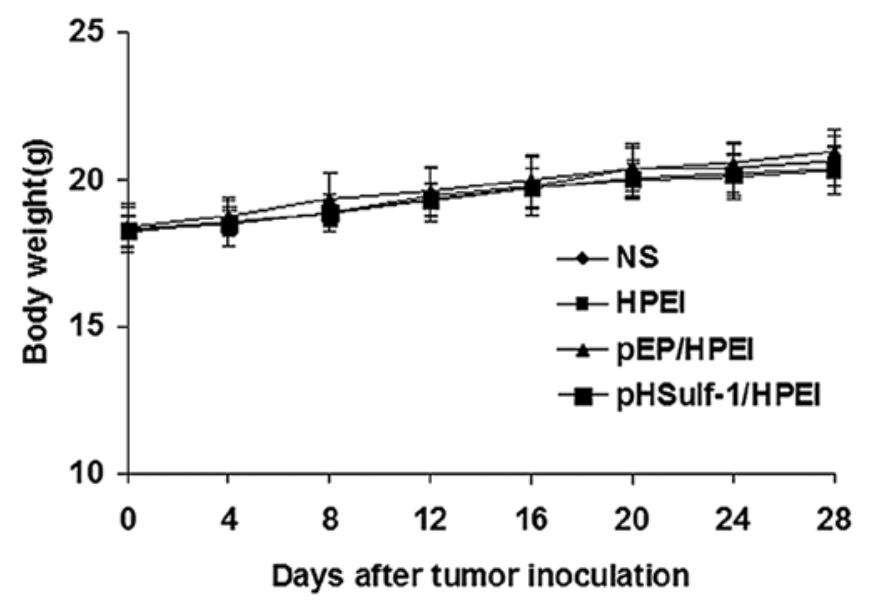

Figure 6. Lack of toxicity-dependent weight loss in tumor-bearing mice treated with pHSulf-1/HPEI complexes. There were no significant differences in weight among the four groups $(\mathrm{P}>0.05)$. The data are expressed as the means $\pm \mathrm{SD} ; \mathrm{n}=6$ for each group.

or cachexia, was monitored every four days. No apparent differences in body weight were found among the four groups (Fig. 6) and no gross changes such as ruffling of fur and changes in behavior occurred in the pHSulf-1/HPEI complex treated group. Additionally, no obvious pathological changes of important organs were observed by HE histological staining analysis.

\section{Discussion}

Previous studies have reported HSulf- 1 could play a key role in regulating tumor cell growth and angiogenesis. In the present study, we have demonstrated that a recombinant plasmid DNA encoding HSulf-1 delivered by biodegradable cationic HPEI nanogels can efficiently inhibit intraperitoneal xenograft growth of human ovarian cancer, without apparent systemic toxic effects. Furthermore, this study provides evidence of the possible mechanism that HSulf-1 possesses antitumor capability against human ovarian cancer in vivo by way of reducing angiogenesis, decreasing cell proliferation and inducing apoptosis, and opens novel ways for future investigations of this approach.

HSulf-1, an extracellular arylsulfatase which selectively removes 6-O-sulfate from heparan sulfate is down-regulated in the majority $(\sim 75 \%)$ of tumor tissues originated from ovarian cancer patients (6). Loss of HSulf-1 up-regulates the signaling of a variety of heparin-binding growth factors and cytokines, such as FGF-2, HGF and $\mathrm{VEGF}_{165}$, resulting in tumor cell proliferation and resistance to drug-induced apoptosis in vitro (6-9). In addition, forced expression of HSulf-1 can inhibit tumor growth, tumor angiogenesis and enhance drug-induced apoptosis in vivo (10-12). The role of HSulf-1 in growth factor signaling and its effects on human tumorigenesis are under intensive investigation. Therefore, we constructed a plasmid DNA expressing HSulf-1 as a novel antitumor agent and investigated its efficacy in an intraperitoneal xenograft model of human ovarian cancer in nude mice.

So far, many functional genes related with various tumor types have been identified, but the clinical application of gene therapy is limited mainly due to lack of safe and efficient gene delivery technologies. Non-viral gene vectors have many advantages over viral vectors. Most non-viral gene vectors have no limitation to the size of transferred DNA molecules, low immunogenicity and they are easier and cheaper to manufacture than viral vectors (17-19). However, the toxicity is still an obstacle to the application of non-viral vectors in gene therapy (33). As a well-known cationic polymer, polyethyleneimine (PEI) has been proven to be effective for gene transfer due to its condensation of DNA, which facilitates endocytosis, and its 'proton sponger' quality, which can avoid 
the DNA from endosomal disruption $(20,27)$. However, PEI is not biodegradable and has a shortcoming: that is, improvement of transfection efficiency is accompanied with increased cytotoxicity and both transfection efficiency and cytotoxicity increase with its chain length. Furthermore, PEI can induce obvious aggregation of erythrocytes and hemolysis $(20,21)$. To overcome this drawback, in our previous study, the low molecular weight PEI was chemically conjugated into biodegradable cationic nanogels by heparin and then the prepared HPEI nanogels were used to deliver the plasmid as a novel gene delivery vector (27). Moreover, HPEI nanogels showed lower cytotoxicity, better blood compatibility, stability in vitro and could be degraded into low molecular weight PEI followed by excretion through urine quickly in vivo. Because of these properties of HPEI nanogels, in the present study, we used HPEI nanogels as the gene transfer vector to further investigate the efficacy and safety of this approach.

Our data indicated that pHSulf- 1 could be efficiently transfected into SKOV3 ovarian cancer cells by HPEI nanogels in vitro and in vivo, without emerging apparent cytotoxicity and systemic toxic effects. Expression of HSulf-1 in vitro and in vivo was verified by RT-PCR and Western blot analysis. Intraperitoneal injection of pHSulf-1/HPEI complexes efficiently suppressed tumor growth and invasion compared with control agents, similar to that observed by Dai et al (11) in myeloma model and Narita et al (12) in breast carcinoma model. We made several observations to elucidate the possible antitumor mechanism of pHSulf-1 in vivo. CD31 immunostaining and alginate-encapsulate tumor cell assay showed that pHSulf-1 resulted in apparent decrease in tumor vessel formation compared with control therapies. These results were in agreement with a previous report that HSulf-1 could inhibit angiogenesis in vivo (12). This indicates that the intraperitoneal tumor might be exposed to a poor supply of oxygen and nutrients, which inhibits tumor growth consistent with a universally accepted concept that the growth of solid tumor is dependent on their capability to induce tumor angiogenesis (34). The growth, persistence and metastases of most solid tumors like ovarian cancer are angiogenesis-dependent, and angiogenesis is regulated by the balance between various proangiogenic and anti-angiogenic factors $(35,36)$. Because heparan sulfate can interact with considerable numbers of these factors to enhance or inhibit their activities, HSulf-1 may be a potential regulatory role in this complex procedure of pathological angiogenesis. Angiogenesis constitutes an important point in the control of cancer progression, and one step in the malignant progression of cancer is an angiogenic switch from a non-angiogenic phenotype. This involves down-regulation of tumor suppressor genes, overexpression of oncogenes, and secretion of a variety of growth factors that may act by autocrine and paracrine signal pathways (37). Whether HSulf-1 is one of the markers of this angiogenic switch or not is currently undergoing active investigation.

TUNEL assay showed pHSulf-1 led to abundant apoptosis of tumor cells compared with control agents, consistent with that observed by Narita et al (12) in breast carcinoma model. One of the crucial therapeutic strategies against cancer is to induce tumor cell apoptosis. Previous studies have shown that the presence of HSulf-1 in some type of cancer cells sensitizes these cells to conventionally used chemotherapeutic drugs such as cisplatin and paclitaxel, and enhances drug-induced apoptosis $(6-8,10)$. The combination therapy that combines suppression of angiogenesis with traditional chemotherapeutic drugs may exert a more noticeable effect of reducing tumor burden and metastases in vivo (38). Moreover, the result of Ki-67 immunostaining suggested that the expression of HSulf-1 could inhibit ovarian cancer cell proliferation, in agreement with previous reports indicating that HSulf-1 expression down-regulated heparin-binding growth factor signaling, consequently resulting in decreased growth rate of several cancer cell lines $(6-8,11,12)$. Due to these observations that the presence of HSulf-1 can inhibit angiogenesis, decrease cell proliferation and induce tumor cell apoptosis in vivo, we hope that the therapeutic effect would be more beneficial through combination gene therapy of pHSulf-1/HPEI complexes with traditional chemotherapeutic drugs.

In conclusion, pHSulf-1 could be efficiently transfected into ovarian cancer cells by HPEI nanogels in vitro and in vivo, and HPEI nanogels could serve as an effective and safe non-viral gene vector. The application of pHSulf-1/HPEI complexes efficiently inhibited angiogenesis, decreased cell proliferation and induced apoptosis, thereby reducing tumor burden in intraperitoneal carcinomatosis model of human ovarian cancer. Treating ovarian cancer by way of pHSulf-1 delivered by HPEI nanogels might become a new and promising gene therapy protocol.

\section{Acknowledgements}

This study was supported by the National Key Basic Research Program (973 Program) of China (2010CB529905 and 2011CB910703).

\section{References}

1. Morimoto-Tomita M, Uchimura K, Werb Z, Hemmerich S and Rosen SD: Cloning and characterization of two extracellular heparin-degrading endosulfatases in mice and humans. J Biol Chem 277: 49175-49185, 2002.

2. Dhoot GK, Gustafsson MK, Ai X, Sun W, Standiford DM and Emerson CP Jr: Regulation of Wnt signaling and embryo patterning by an extracellular sulfatase. Science 293: 1663-1666, 2001.

3. Selva EM and Perrimon N: Role of heparan sulfate proteoglycans in cell signaling and cancer. Adv Cancer Res 83: 67-80, 2001

4. Lin X: Functions of heparan sulfate proteoglycans in cell signaling during development. Development 131: 6009-6021, 2004.

5. Kirkpatrick CA and Selleck SB: Heparan sulfate proteoglycans at a glance. J Cell Sci 120: 1829-1832, 2007.

6. Lai JP, Chien J, Staub J, Avula R, Greene EL, Matthews TA, Smith DI, Kaufmann SH, Roberts LR and Shridhar V: Loss of HSulf-1 up-regulates heparin-binding growth factor signaling in cancer. J Biol Chem 278: 23107-23117, 2003.

7. Lai JP, Chien J, Strome SE, Staub J, Montoya DP, Greene EL, Smith DI, Roberts LR and Shridhar V: HSulf-1 modulates HGF-mediated tumor cell invasion and signaling in head and neck squamous carcinoma. Oncogene 23: 1439-1447, 2004.

8. Lai JP, Chien JR, Moser DR, Staub JK, Aderca I, Montoya DP, Matthews TA, Nagorney DM, Cunningham JM, Smith DI, Greene EL, Shridhar V and Roberts LR: hSulf1 sulfatase promotes apoptosis of hepatocellular cancer cells by decreasing heparinbinding growth factor signaling. Gastroenterology 126: 231-248, 2004.

9. Staub J, Chien J, Pan Y, Qian X, Narita K, Aletti G, Roberts LR, Molina J and Shridhar V: Epigenetic silencing of HSulf-1 in ovarian cancer: implications in chemoresistance. Oncogene 26: 4969-4978, 2007.

10. Liu P, Khurana A, Rattan R, Kalloger S, Dowdy S, Gilks B and Shridhar V: Regulation of HSulf-1 expression by variant hepatic nuclear factor 1 in ovarian cancer. Cancer Res 69: 4843-4850, 2009. 
11. Dai Y, Yang Y, MacLeod V, Yue X, Rapraeger AC, Shriver Z, Venkataraman G, Sasisekharan R and Sanderson RD: HSulf-1 and HSulf-2 are potent inhibitors of myeloma tumor growth in vivo. J Biol Chem 280: 40066-40073, 2005.

12. Narita K, Staub J, Chien J, Meyer K, Bauer M, Friedl A, Ramakrishnan S and Shridhar V: HSulf-1 inhibits angiogenesis and tumorigenesis in vivo. Cancer Res 66: 6025-6032, 2006.

13. Li J, Kleeff J, Abiatari I, Kayed H, Giese NA, Felix K, Giese T, Buchler MW and Friess H: Enhanced levels of HSulf-1 interfere with heparin-binding growth factor signaling in pancreatic cancer. Mol Cancer 4: 1-14, 2005.

14. Nawroth R, van Zante A, Cervantes S, McManus M, Hebrok M and Rosen SD: Extracellular sulfatases, elements of the wnt signaling pathway, positively regulate growth and tumorigenicity of human pancreatic cancer cells. PLoS One 2: e392, 2007.

15. Edelstein ML, Abedi MR, Wixon J and Edelstein RM: Gene therapy clinical trials worldwide 1989-2004 - an overview. J Gene Med 6: 597-602, 2004.

16. Anderson DG, Peng W, Akinc A, Hossain N, Kohn A, Padera R, Langer R and Sawicki JA: A polymer library approach to suicide gene therapy for cancer. Proc Natl Acad Sci USA 101: 16028-16033, 2004.

17. Cavazzana-Calvo M, Thrasher A and Mavilio F: The future of gene therapy. Nature 427: 779-781, 2004.

18. Ferber D: Gene therapy: safer and virus-free? Science 294 $1638-1642,2001$.

19. Glover DJ, Lipps HJ and Jans DA: Towards safe, non-viral therapeutic gene expression in humans. Nat Rev Genet 6: 299-310, 2005.

20. Neu M, Fischer D and Kissel T: Recent advances in rational gene transfer vector design based on poly(ethyleneimine) and its derivatives. J Gene Med 7: 992-1009, 2005.

21. Kunath K, von Harpe A, Fischer D, Petersen H, Bickel U, Voigt K and Kissel T: Low-molecular-weight polyethyleneimine as a non-viral vector for DNA delivery: comparison of physicochemical properties, transfection efficiency and in vivo distribution with high-molecular weight polyethyleneimine. J Controlled Release 89: 113-125, 2003.

22. Wen Y, Pan S, Luo X, Zhang X, Zhang W and Feng M: A biodegradable low molecular weight polyethyleneimine derivative as low toxicity and efficient gene vector. Bioconjugate Chem 20: 322-332, 2009

23. Gosselin MA, Guo W and Lee RJ: Efficient gene transfer using reversibly cross-linked low molecular weight polyethyleneimine. Bioconjugate Chem 12: 989-994, 2001.

24. Ahn CH, Chae SY, Bae YH and Kim SW: Biodegradable poly(ethyleneimine) for plasmid DNA delivery. J Controlled Release 80: 273-282, 2002.
25. Forrest ML, Koerber JT and Pack DW: A degradable polyethyleneimine derivative with low toxicity for highly efficient gene delivery. Bioconjugate Chem 14: 934-940, 2003.

26. Jeon O, Yang HS, Lee TJ and Kim BS: Heparin-conjugated polyethyleneimine for gene delivery. J Controlled Release 132: 236-242, 2008.

27. Gou ML, Men K, Zhang J, Li YH, Song J, Luo S, Shi HS, Wen YJ, Guo G, Huang MJ, Zhao X, Qian ZY and Wei YQ: Efficient inhibition of C-26 colon carcinoma by VSVMP gene delivered by biodegradable cationic nanogel derived from polyethyleneimine. ACS Nano 4: 5573-5584, 2010.

28. Jemal A, Siegel R, Ward E, Hao Y, Xu J and Thun MJ: Cancer statistics, 2009. CA Cancer J Clin 59: 225-249, 2009.

29. Ozols RF: Progress in ovarian cancer: an overview and perspective. EJC (Suppl) 1: 43-55, 2003.

30. Lin XJ, Chen XC, Wang L, Wei YQ, Kan B, Wen YJ, He X and Zhao X: Dynamic progression of an intraperitoneal xenograft model of human ovarian cancer and its potential for preclinical trials. J Exp Clin Cancer Res 26: 467-474, 2007.

31. Hoffmann J, Schirner M, Menrad A and Schneider MR: A highlysensitive model for quantification of in vivo tumor angiogenesis induced byalginate-encapsulated tumor cells. Cancer Res 57: 3847-3851, 1997.

32. He QM, Wei YQ, Tian L, Zhao X, Su JM, Yang L, Lu Y, Kan B, Lou YY, Huang MJ, Xiao F, Liu JY, Hu B, Luo F, Jiang Y, Wen YJ, Deng HX, Li J, Niu T and Yang JL: Inhibition of tumor growth with a vaccine based on xenogeneic homologous fibroblast growth factor receptor-1 in mice. J Biol Chem 278: 21831-21836, 2003.

33. Lv H, Zhang S, Wang B, Cui S and Yan J: Toxicity of cationic lipids and cationic polymers in gene delivery. J Controlled Release 114: 100-109, 2006.

34. Neri D and Bicknell R: Tumour vascular targeting. Nat Rev Cancer 5: 436-446, 2005.

35. Folkman J: What is the evidence that tumors are angiogenesisdependent? J Natl Cancer Inst 82: 4-6, 1990.

36. Carmeliet $P$ and Jain RK: Angiogenesis in cancer and other diseases. Nature 407: 249-257, 2000

37. Folkman J: Role of angiogenesis in tumor growth and metastasis. Semin Oncol 29: 15-18, 2002.

38. Hu L, Hofmann J, Zaloudek C, Ferrara N, Hamilton T and Jaffe RB: Vascular endothelial growth factor immunoneutralization plus Paclitaxel markedly reduces tumor burden and ascites in athymic mouse model of ovarian cancer. Am J Pathol 161: $1917-1924,2002$ 